\title{
An Adaptive Partial Differential Equation for Noise Removal
}

\author{
Chao Shen \\ Xi'an University of Posts and \\ Telecommunications, \\ School of Communication and \\ Information Engineering, \\ Xi'an, China \\ email:18792853024@163.com
}

\author{
Changxing Li \\ Xi'an University of Posts and \\ Telecommunications, \\ School of Science, \\ Xi’an, China \\ email:13072957879@163.com
}

\author{
Pingping Wang \\ Xi'an University of Posts and \\ Telecommunications \\ School of Communication and \\ Information Engineering \\ Xi'an, China \\ e-mail: 446355436@qq.com
}

\begin{abstract}
In order to preserve the image edge feature while removing the noise, an adaptive joint denoising model has been proposed which jointed the second-order and fourth-order partial differential equations (PDE) model. Firstly, improving the traditional fourth-order PDE, separating its diffusion along two orthogonal directions, and then acquiring the separating coefficient fourth-order PDE model. Secondly, this improved fourth-order model combined with the second-order inhomogeneous mean curvature diffusion model, meanwhile, setting the adaptive weights coefficient between second-order and fourth-order models. Finally, the image fidelity term has been added to adaptive joint model. The simulation results show that the adaptive joint model can alleviate blocky and speckle effect effectively, and it has better quantitative results.
\end{abstract}

Keywords- adaptive denoising; PDE; separating coefficient component

\section{INTRODUCTION}

The purpose of image denoising is to preserve edge as far as possible while remove noise, making the resulting images approximate the ideal image. The searchers found that processing image in spatial domain can better preserve geometrical characteristics when removing noise, this is a significant feature of PDE. And PDE has mature numerical calculation methods, the better recovery quality, equation stability and other characteristics. In 1990, proposing anisotropic diffusion P-M model, and then, researchers put forward anisotropic diffusion second-order PDE model [1-3], total variation TV model [4-7], image level set geometric heat flow equations [8], anisotropic diffusion fourth-order PDE model [9-10] and fractional-order PDE model [11].

Second-order PDE uses increasing function regard to gradient operator absolute value as integrand of energy functional. This type PDE can better preserve edges when removing the noise, but its resulting image exists serious blocky effect. In computer processing, the edge of blocky may be considered as the edge of ideal image, but this edge does not exist in ideal image. Furth-order PDE model uses increasing function regard to Laplacian operator absolute value as integrand of energy functional. Its resulting image's smoothness is better than second-order PDE, but it introduces speckle effect simultaneously, and because Laplacian operator cannot determine edges, this will lead to a phenomenon that fourth-order PDE model blurs edge information.

For above reasons, firstly, we improved the traditional fourth-order PEDS model that separating the diffusion along two orthogonal directions, so that this diffusion along the respective direction will not affect by diffusion along orthogonal direction, this model is called separating coefficient fourth-order PDE (SCFO) model. Secondly, we combined SCFO model and second-order inhomogeneous mean curvature diffusion (MCD) model [12], and setting this two models can change the weights adaptively in new model, through taking advantage of the fourth-order model's better image smoothing effects and the second-order model's better edge preserving effects to achieve this effect that preserving the edge while removing the noise. Finally, we added the image fidelity term $\left(u-u_{0}\right)$ to new model, this is conducive to better preserve the image edge information. We called this new PDE model as adaptive joint model (AJ model). The rest of this paper is organized as follows. The second section introduces MCD model and the traditional fourth-order PDE model. The third section describes AJ mode. The fourth section introduces the difference scheme of AJ model. The fifth section describes numerical experiment and the conclusion is drawn in sixth section.

\section{The TRAditional Model}

\section{A. MCD model}

In image processing, we hope to accelerate the diffusion to remove noise in planar area and weaken the diffusion to preserve edge in boundary area. MCD model is proposed based on this idea, its corresponding scalar PDE as follows.

$$
\frac{\partial u}{\partial t}=\frac{\partial}{\partial x}\left(\frac{u_{x}}{\sqrt{u_{x}^{2}+u_{y}^{2}+1}}\right)+\frac{\partial}{\partial y}\left(\frac{u_{y}}{\sqrt{u_{x}^{2}+u_{y}^{2}+1}}\right)=H
$$

Where $H$ is an average value of image intensity function along two orthogonal directions normal curvature.

Second-order PDE uses a piecewise level image to approximate ideal image, so as a type of second-order PDE model, MCD model exists obvious blocky effect inevitably. 


\section{B. The traditional fourth-order PDE model}

The traditional fourth-order PDS model's energy functional shows as follows.

$$
\frac{\partial u}{\partial t}=-\Delta[c(|\Delta u|) \Delta u]
$$

Where $c(|\Delta u|)=\frac{f^{\prime}(|\Delta u|)}{|\Delta u|}$, it is a positive and decreasing function regard to argument $|\Delta u|, f(|\Delta u|)$ is a positive and increasing function of argument $|\Delta u|$.

The traditional fourth-order PDE model uses a piecewise planar image to approximate ideal image, it can alleviate blocky effect effectively which generated by second-order PDE, but it is influenced by speckle effect. Comparing the intensity value between speckle area and its neighborhood, we found that their values are greatly different, but the coefficient $c(|\Delta u|)$ is a positive and decreasing function, so this diffusion rarely occurs in this speckle area, so this speckle area will preserve in diffusion procedure. On the other hand, when $u_{x x} \approx-u_{y y}$ and $\left|u_{x x}\right|>>0$ and $\left|u_{y y}\right|>>0$, then $|\Delta u| \approx 0$, so diffusion coefficient $c(|\Delta u|)>>0$, then it will produce the phenomenon that the diffusion is more in image large undulating area. In resulting image, it shows that the image edge will be destroyed.

Through above analysis, we can see that second-order PDE model can better preserve image edge ,but it easily introduces blocky effect; Fourth-order PDE model can better smooth image, but it easily put speckle effect into resulting image and it blurs image edge. Therefore, we improve the traditional fourth-order PDE model to get SCFO model, and then it combines with second-order MCD model to propose AJ model.

\section{AJ MODEL}

\section{A. SCFO model}

We separate the image Laplacian operator to process, and define following energy functional.

$$
E(u)=\int_{\Omega}\left[f\left(\left|u_{x x}\right|\right)+f\left(\left|u_{y y}\right|\right)\right] d x d y
$$

Where $f(s)$ is a positive and increasing function regard to argument $s$. Its gradient descent flow equation shows as follow.

$$
\frac{\partial u}{\partial t}=-\left[\frac{\partial^{2}}{\partial x^{2}}\left(c\left(\left|u_{x x}\right|\right) u_{x x}\right)+\frac{\partial^{2}}{\partial y^{2}}\left(c\left(\left|u_{y y}\right|\right) u_{y y}\right)\right]
$$

Where $c(s)=\frac{f^{\prime}(s)}{s}$, it is a positive and decreasing function regard to argument $s$.

Form Eq. (4), we can see that the diffusion coefficient $c\left(\left|u_{x x}\right|\right)$ along $x$ only is related to $\left|u_{x x}\right|$, and nothing to do with $\left|u_{y y}\right|$; the one along $y$ is in a similar way. Then the diffusion along the respective directions will not be influenced by diffusion along another orthogonal direction, the diffusion procedure will be more precise, this can better preserve edge while removing noise. SCFO model can better reduce speckle, but it cannot eliminate speckle. Therefore, we consider to combine MCD model and SCFO model to process the degrade image, this leads to AJ model.

\section{B. AJ model}

We combined SCFO model, MCD model and image fidelity term, and setting the weights as adaptive function form between SCFO and MCD model. Therefore, the gradient descent flow equation as follows.

$$
\frac{\partial u}{\partial t}=-w F+(1-w) H-\lambda_{2}\left(u-u_{0}\right)
$$

Where

$$
w=\frac{\lambda_{1}\left(\left|u_{x x}\right|+\left|u_{y y}\right|\right)}{|\nabla u|+\left|u_{x x}\right|+\left|u_{y y}\right|}
$$

$F=\frac{\partial^{2}}{\partial x^{2}}\left(c\left(\left|u_{x x}\right|\right) u_{x x}\right)+\frac{\partial^{2}}{\partial y^{2}}\left(c\left(\left|u_{y y}\right|\right) u_{y y}\right)$, and $H$ is shown in Eq. (1), $\lambda_{1}$ and $\lambda_{2}$ are constants.

In Eq. (5), the weights coefficient $w$ can adaptively adjust the weights of SCFO model and MCD model. When $w=1$, AJ model degenerates SCFO model which combines with image fidelity term; When $w=0$, AJ model degenerates MCD model which combines with image fidelity term. Thus, AJ model can adaptively adjust weights of SCFO model and MCD model according to image actual situation, so AJ model has fourth-order PDE model's better image smoothing property as well as second-order model's better edge preserving property, this model can get better resulting image.

\section{DIFFERENCE SCHEME}

We use iteration method to solve Eq. (5). Assuming that image size is $I \times J$, iterative time step is $\Delta t$, the spatial grid length is $h$, then time $t$ and spatial length $x, y$ are quantized as follows.

$$
\begin{aligned}
t & =n \Delta t, n=0,1,2 \cdots \\
x & =i h, i=0,1,2, \cdots, I \\
y & =j h, j=0,1,2, \cdots, J
\end{aligned}
$$

We use symmetry boundary condition $\frac{\partial u}{\partial n}=0$, where $n$ represents unit normal vector at image boundary. This condition can be quantized as follows.

$$
\begin{aligned}
& u_{-1, j}^{n}=u_{0, j}^{n}, u_{I, j}^{n}=u_{I+1, j}^{n}, j=1,2, \cdots, J \\
& u_{i,-1}^{n}=u_{i, 0}^{n}, u_{i, J}^{n}=u_{i, J+1}^{n}, i=1,2, \cdots, I
\end{aligned}
$$

We use slightest center difference to quantized image's first-order and second-order derivate.

$$
\frac{\partial u_{i, j}^{n}}{\partial x}=\frac{u_{i+1, j}^{n}-u_{i-1, j}^{n}}{2 h}, \frac{\partial^{2} u_{i, j}^{n}}{\partial x^{2}}=\frac{u_{i+1, j}^{n}+u_{i-1, j}^{n}-2 u_{i, j}^{n}}{h^{2}}
$$

The one along $y$ is similar to this. Then 


$$
w=\frac{\lambda_{1}\left(\left|\frac{\partial^{2} u_{i, j}^{n}}{\partial x^{2}}\right|+\left|\frac{\partial^{2} u_{i, j}^{n}}{\partial y^{2}}\right|\right)}{\sqrt{\left(\frac{\partial u_{i, j}^{n}}{\partial x}\right)^{2}+\left(\frac{\partial u_{i, j}^{n}}{\partial y}\right)^{2}}+\left|\frac{\partial^{2} u_{i, j}^{n}}{\partial x^{2}}\right|+\left|\frac{\partial^{2} u_{i, j}^{n}}{\partial y^{2}}\right|} .
$$

$$
g_{1}=c\left(\left|u_{x x}\right|\right) u_{x x} \quad, \quad g_{2}=c\left(\left|u_{y y}\right|\right) u_{y y}, \quad g_{3}=\frac{u_{x}}{\sqrt{u_{x}^{2}+u_{y}^{2}+1}} \text {, }
$$$$
g_{4}=\frac{u_{y}}{\sqrt{u_{x}^{2}+u_{y}^{2}+1}} \text {, then }
$$$$
F_{i, j}^{n}=\frac{\partial^{2} g_{1 i, j}^{n}}{\partial x^{2}}+\frac{\partial^{2} g_{2 i, j}^{n}}{\partial y^{2}}, H_{i, j}^{n}=\frac{\partial g_{3 i, j}^{n}}{\partial x}+\frac{\partial g_{4 i, j}^{n}}{\partial y}
$$

At last, the difference equation of $\mathrm{AJ}$ model shows as follows.

$$
\begin{aligned}
u_{i, j}^{n+1}=u_{i, j}^{n}+\Delta t\left(-w_{i, j}^{n} F_{i, j}^{n}+\left(1-w_{i, j}^{n}\right) H_{i, j}^{n}\right. & \left.-\lambda_{2}\left(u_{i, j}^{n}-u_{0 i, j}\right)\right)
\end{aligned}
$$

\section{NUMERICAL EXPERIMENT}

In experiment, through adding additive Gaussian noise to ideal image to get degrade image, the arguments of Gaussian noise are $m=0$ and $\sigma=20$. The diffusion coefficient form of SCFO model is $c(s)=\frac{1}{s}$ in AJ model. The argument $\lambda_{1}$ of weights coefficient is $\lambda_{1}=0.5$. The argument $\lambda_{2}$ of fidelity term is $\lambda_{2}=0.01$. The time step and spatial grid length set $\Delta t=0.25$ and $h=1$ respectively.

We use image peak signal-to-noise ratio (PSNR), image signal-to-noise (SNR) and cross-entropy $(\mathrm{H})$ as quantitative criteria of result comparison. They defined as follows.

$$
\begin{aligned}
P S N R & =10 \log _{10}\left(\frac{255 \times 255}{M S E}\right) \\
S N R & =10 \log _{10}\left(\frac{R M S E}{M S E}\right) \\
H & =-\sum_{i=0}^{255} p_{i} \log \left(q_{i}\right)
\end{aligned}
$$

Where RMSE and MSE represent image and image's noise mean square error respectively, $p_{i}$ and $q_{i}$ represent probability of two image intensity class $i$ respectively.

We applied MCD model, XZS model[13] and AJ model to LENA image, its results as follows.

TABLE I. COMPARISON OF DENOISING MODELS

\begin{tabular}{|c|c|c|c|}
\hline \multirow{2}{*}{} & \multicolumn{3}{|c|}{} \\
\cline { 2 - 4 } PSNR & MCD model & XZS model & AJ model \\
\hline SNR & 29.1959 & 29.1929 & 29.6251 \\
\hline H & 14.3968 & 14.3515 & 14.8324 \\
\hline
\end{tabular}

From the quantitative result in TABLE 1, we can see that AJ model's PSNR, SNR are better than MCD and XZS model, and this three models' $\mathrm{H}$ are almost equivalent. From Fig. 1,we can see that MCD model's resulting image still exists obvious blocky effect, and XZS model's resulting image still exists obvious speckle effect, however, AJ model can alleviate blocky and speckle effect simultaneously, and resulting image is more natural and rarely emerge wrong blocky edge.

We show the results as follows which uses LADY image to demonstrate AJ model.

TABLE II. COMPARISON OF DENOISING MODELS

\begin{tabular}{|c|c|c|c|}
\hline \multirow{2}{*}{} & \multicolumn{3}{|c|}{} \\
\cline { 2 - 4 } & MCD model & XZS model & AJ model \\
\hline PSNR & 31.5366 & 31.5706 & 32.0639 \\
\hline SNR & 19.8419 & 19.8373 & 20.3417 \\
\hline H & 5.1795 & 5.1844 & 5.1832 \\
\hline
\end{tabular}

From TABLE 2 and Fig. 2, we can see that the results also verified validity of AJ model.

\section{CONCLUSION}

In this paper, firstly, we improved the traditional fourthorder PDE model to get SCFO model. Then we combine second-order MCD, fourth-order SCFO model which sets adaptive weights between these two models. Finally, we added image fidelity term to get AJ model. AJ model can change weights according to actual image situation to obtain better resulting image. The experiment results show that AJ model can obtain better quantitative results and natural and accurate resulting image.

\section{REFERENCES}

[1] Wang M J, Deng S X. Image Restoration Model of PDE Variation. IEEE Information and Computer Science, 2009, 2: 184-187

[2] Wu J, Tang C. PDE-Based Random-Valued Impulse Noise Removal Based on New Class of Controlling Function. IEEE Transactions on Image Processing, 2011, 20(9): 2428-2438

[3] Rafsanjani H K, Saryazdi S, Zeinali M H, et al. A denoising algorithm based on PDE variational approach using PM potential. IEEE Machine Cision and Image Processing, 2011, 1-5

[4] Rafsanjani H K, Saryazdi S, Zeinali M H, et al. A denoising algorithm based on PDE variational approach using PM potential. IEEE Machine Cision and Image Processing, 2011, 1-5

[5] Shi M Z, Xu T F. Adaptive Total Variation Image Restoration Coupling with a Gradient Fidelity Term. IEEE Image and Signal Processing, 2011,2: 821-825

[6] Fazli S, Bouzari H, Pour H M. Complex PDE image denoising based on Partial Swarm Optimization. IEEE International Congress on Ultra Modern Telecommunications and Control Systems and Workshops, 2010, 364-370

[7] Bae E, Shi J, Tai X C. Graph Cuts for Curvature Based Image Denoising. IEEE Transactions on Image Processing, 2011, 20(5):1199-1210

[8] Lee S H, Seo J K. Noise removal with Gauss curvature-driven diffusion. IEEE Transactions on Image Processing, 2005, 14 (7): 904-909 
[9] You Y L, Kaveh M. Fourth-order partial differential equations for noise removal. IEEE Transactions on Image Processing, 2000, 9(10): 1723-1730

[10] Kim S, Lim H. Fourth-order partial differential equations for effective image denoising. Electronic Journal of Differential Equations, 2009, 107-121

[11] Bai J, Feng X C. Fractional-order Anisotropic Diffusion for Image Denoising. IEEE Transactions on Image Processing, 2007, 16(10): 2492-2502
[12] El-Fallah A I, Ford G E. Mean curvature evolution and surface area scaling in image filtering. IEEE Transactions on Image Processing, 1997, 6(5): 750-753

[13] Liu X-L , Ying Z-W, Qiu S-F. A fourth-order partial differential equations method of noise removal. IEEE Image and Signal Processing, 2011, 2: 641-645

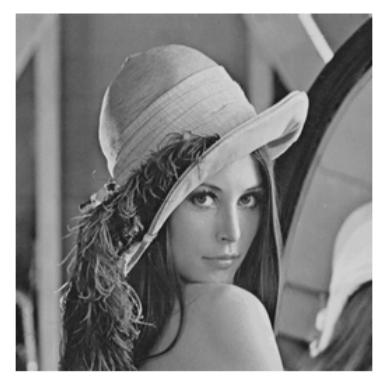

(a)Ideal image

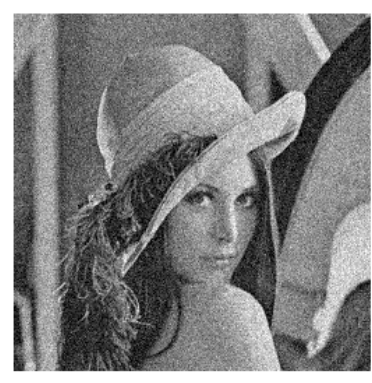

(b)Degrade image

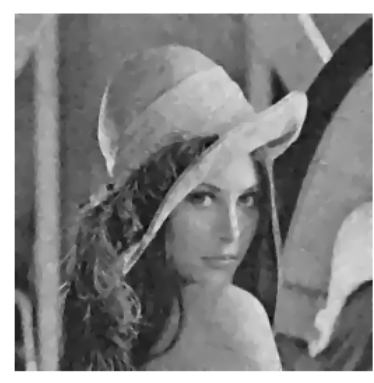

(c)MCD model's result
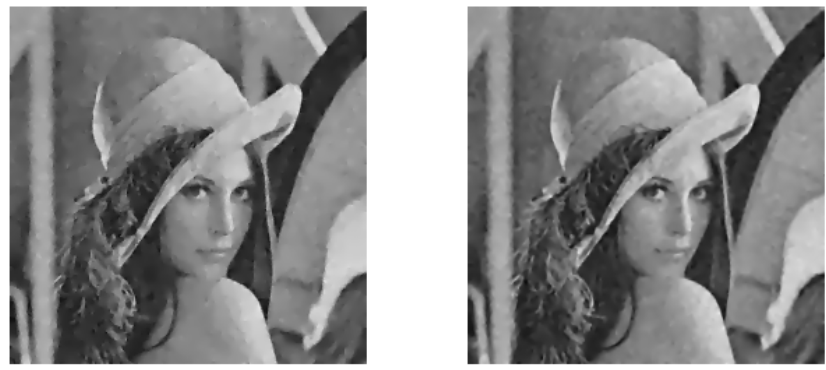

(d)XZS model's result

(e)AJ model's result

Figure 1. Resulting images of Lena.

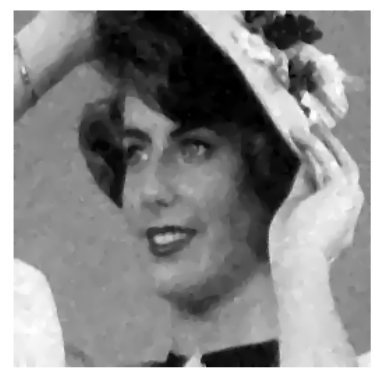

(a)MCD model's result

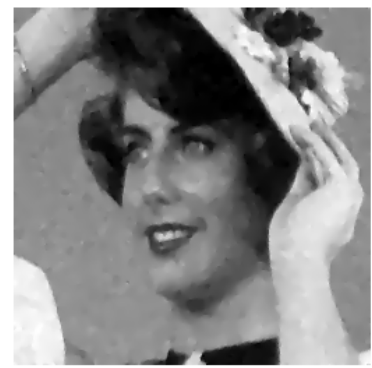

(b)XZS model's result

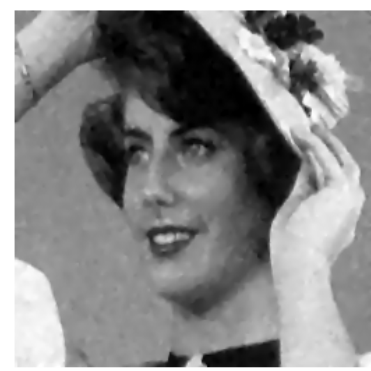

(c)AJ model's result

Figure 2. Resulting images of Lady. 